Ann. Zootech., Ig6r, 10 (3), 229-232.

\title{
OBSERVATIONS SUR L'APPLICATION PRATIQUE DE L'INSÉMINATION ARTIFICIELLE CHEZ LES BOVINS, LES BUFFLES, LES CHÈVRES A IZATNAGAR (INDE).
}

\author{
G. SINGH
}

Division of animal Genetics, Indian veterinary Research Institute, Izatnagar (Inde).

Il existe très peu de bons Taureaux aux Indes. La proportion de I sur 250 a été avancée (MAKRIJANI, I947). Cette situation incitait à introduire sur une grande échelle l'insémination artificielle dans l'espèce bovine.

Cependant les études systématiques sur l'emploi de l'insémination artificielle dans les conditions d'élevage des Indes ne débutèrent qu'en I942 à l'Institut de recherches vétérinaires d'Izatnagar.

L'application de l'insémination artificielle est principalement du ressort du Service de la reproduction de l'État.

Si la physiopathologie de la reproduction des bovins est bien connue, il existe moins d'informations sur les Chèvres, et très peu sur les Buffles.

Peu d'observations ont été faites sur les caprins, bien que cette espèce soit utilisée aux Indes à deux fins : le lait est consommé spécialement par les familles pauvres et la viande est consommée par la majorité des non-végétariens qui la préfèrent au mouton. Aussi davantage de mâles sont nécessaires pour satisfaire la demande de viande et davantage de femelles pour produire du lait.

L'introduction de l'insémination artificielle pourrait permettre d'améliorer les productions simultanément.

Dans cette note, nous avons voulu présenter les résultats de l'insémination artificielle dans ces 3 espèces au cours des essais effectués pendant de nombreuses années à Izatnagar.

\section{BOVINS}

Nombre d'inséminations et taux de conception. - L'étude porte sur 6768 inséminations effectuées par le centre d'insémination d'Izatnagar de I946 à I958. I,es taux de fécondité sont basés sur les vélages réels. Car dans les conditions difficiles de la pratique, il est impossible d'estimer avec une précision satisfaisante le taux de conception sur la base des non-retours. Le taux général moyen de conception a été de 53,2 p. IOO.

Variations saisonnières $d u$ taux de conception. - Les Vaches viennent en chaleur tout au long de l'année et il n'a été remarqué aucune influence saisonnière. 
Ce qui est en accord avec la majorité des chercheurs (ERB, WILibur, et HilTon, I940 ; Erb, Wilbur et Hiltox; I942 ; Mercier et SAlisbury, i 947 ; Patrick, I952).

Les données relatives à 989 inséminations effectuées de 1956 à I 958 montrent qu'en fonction des saisons le taux de conception a été de 59.3 p. Ioo en automne, 60,0 p. Ioo en hiver, 62,5 p. Ioo au printemps et 66,3 p. Ioo en été. Les différences entre ces résultats ne sont cependant pas significatives. PRAHBU et BHATTACHARYA (I953) ont également constaté qu'il n'y avait pas de variations saisonnières dans le taux de conception d'après des Vaches inséminées artificiellement aux Indes. Pour les bovins européens BURGEss (I953) n'a pas constaté de différence significative selon les mois et les saisons dans le taux de non-retour à 60-9o jours.

Naissances gémellaires. - Deux naissances gémellaires seulement sont rapportées sur 6768 inséminations.

Sex-ratio. - Le sex-ratio calculé sur i 357 vélages a donné 55,3 p. Ioo de mâles. BRANFORD (I957) ayant travaillé sur Vaches laitières indiennes accouplées naturellement avait observé une proportion de mâles de 57,9 p. Ioo. BhatTacharya, PraBHU et ChatTerJeE (I956) ont rapporté un sex-ratio de 50,8 p. Ioo chez des Vaches indiennes accouplées naturellement.

Les variations saisonnières du sex-ratio ne sont pas significatives ce qui est en accord avec les observations de Bhat'tacharia, Prabhu et Chatterjee (ig56).

\section{BUHFLE INDIEN (Bos bubalis)}

Nombre d'inséminations et taux de conception. - Les données obtenues de I946 à I 958 portent sur 5797 inséminations. Le taux moyen de conception pour toutes les inséminations a été de 54,2 p. IoO soit I,88 insémination par gestation.

Pattabiraman (I956), Bhattacharya, Prabhu (I953-54-55) ont trouvé un taux d'insémination pour les Buffles indiens de I,39 à I,59 par gestation. HAFEz (r952-I953) a trouvé que l'accouplement naturel des Buffles égyptiens nécessitait I,39 accouplement par conception.

Il existe une pointe dans le nombre d'insémination en fonction des saisons et les différences de fréquence d'insémination sont statistiquement significatives; elles étaient de 24 p. Ioo en automme, 45,8 p. Ioo en hiver, 22,4 p. Ioo au printemps et 7,75 p. roo en été.

Le nombre maximum de naissances est atteint en septembre (RIFE, 1959). Chez le mâle, l'étude de la variation sexuelle saisonnière a été étudiée par KusHWAHA, Mukherjee et BhatTacharya (I955) et par BhatTacharya (I958). La qualité du sperme est souvent la meilleure en septembre, ce qui correspond approximativement à la période pendant laquelle les femelles entrent en chaleur.

Infuence de variations saisonnières d'activité sexuelle sur le taux de vélage. - Les taux de vélage résultant de I 922 inséminations ne montrent pas de différence significative en faveur d'une influence saisonnière :

57,2 p. Ioo en automne ( 250 sur 437 inséminations) ;

57,3 p. I00 en hiver (530 sur 925 inséminations) ;

52,8 p. IoO au printemps (22I sur 4 I9 inséminations);

52,5 p. Ioo en été (74 sur I4I inséminations).

ASKER et EL-ITRIBY (I958) n'ont également pas trouvé d'influence saisonnière sur la fertilité du Buffle égyptien. 
Sex-ratio. - Sur un total de I 820 naissances de I950 à I958, 1'analyse du sexratio donne $5 \mathrm{I}, 7 \mathrm{p}$. Ioo de mâles, ce qui est en accord avec les observations d'AskER et EL-ITRIBY (I957) qui ont constaté chez le Buffle égyptien accouplé naturellement 52 p. Ioo de mâles. Le sex-ratio ne varie pas en fonction des saisons : 49,7 p. Ioo de mâles en automne, 48,4 p. Ioo en hiver, 52,3 p. Ioo au printemps et 52, I p. Ioo en été (différences non significatives).

\title{
CHÌVRES
}

Nombre d'inséminations et taux de conception. - Au total 685 Chèvres furent inséminées de I950 à 1959 (excepté en I953). Le taux moyen de conception basé sur les mises bas fut de $6 \mathrm{I}, 6 \mathrm{p}$. Ioo soit $\mathrm{I}, 62$ inséminations par gestation.

Variations saisonnières $d u$ taux de conception. - Le taux de conception fut de $60, \mathrm{I}$ p. Ioo en automne, $60,2 \mathrm{p}$. Ioo en hiver, $6 \mathrm{I}, 3 \mathrm{p}$. Ioo au printemps et $65,7 \mathrm{p}$. Ioo en été. Ces différences ne sont pas significatives.

Naissances multiples. - - De 350 naissances, on a enregistré 200 naissances avec un seul jeune soit $57, \mathrm{I}$ p. I00, I 38 avec des jumeaux soit 39,4 p. Ioo, II avec des triplés soit 3, I p. IOO, et une seulement avec quatre jeunes soit 0,3 p. Ioo. La fréquence des naissances multiples paraît donc plus faible que ce qui est généralement rapporté.

C'est ainsi que SETrNski et al. (1956) ont trouvé sur 372 mises bas, 99 produisant des simples soit 26,7 p. I00, I93 produisant des doubles soit 5I,85 p. I00, 67 produisant des triplés soit I8, I p. IOO, I I produisant des quadruplés soit 2,3 p. IOO et 2 , soit 0,54 p. Ioo, des quintuplés.

Sex-ratio. - 55,60 p. Ioo des Chevreaux nés étaient des mâles, valeur en accord avec celle trouvée par Buechi (I957) qui a observé 55,8 p. Ioo de mâles dans trois races de Chèvres à cornes (contre 64,5 p. Ioo dans trois races de Chèvres sans corne). Nous n'avons pas recueilli d'information sur le pourcentage des individus intéressés.

Reçu en septembre 1961.

\author{
SUMMARY \\ PRATICAL STUDY OF ARTIFICIAL INSEMINATION FOR BOVINES, \\ BUFFALOES AND GOATS AT IZATNAGAR (INDIA).
}

This note gives the results of artificial insemination at Izatnagar.

I. Bovines. -6768 inseminations from 1946 to 1958 . Based on calving, the mean conception rate was 53 p. Ioo. No seasonal variation was observed in the sex-ratio and the conception rate.

2. Indian Buffalo (Bos bubalis). - 5797 inseminations from 1946 to 1958 . The mean conception rate was 54.2 p. 100 . The insemination rate varied significantly with the season. But seasonal variations have no influence on the calving rate and the sex-ratio.

3. Geats. - 680 inseminations from 1950 to 1959 . The nean birth ratte was $61.6 \mathrm{p}$. joo. The conception rate is not inlluenced by seasonal variation. 


\section{RÉFÉRENCES BIBLIOGRAPHIQUES}

Askek A. X., E, ITriby A. A., 1957. Calf mortality, abortion, twinning and sex-ratio in exyptian buffalnes Emp. J. Exp. Agric., 25, $15 \mathrm{I}-155$, in Anim. Breed. Abstr., 25, I 25.

Askif $\Lambda$. N., JL ITRIRY A. A., 958 . Frequency of using bulls for service and the distribution of calving in the egyptian buffaloes. Alexandria J. Agric. Res., 6, 25-38, in Anim. Breed. Abstr., 26, 297.

Bhattacinary P., r958. Some aspects of reproduction in Indian farm animals. Presid. ad. Proc., $45 \mathrm{th}$ Indian Sci. Congr., l'art II, pP. 99.

Bhattacharya P., PRABilU S. S., 1953-1955. Field application of artificial insemination in cattle. I, II, III et IV. Indian J. Vel. Sci., 22, I63-I 78;24, 67-77;25, 263-276 ;25, 277-29I.

Bilattacharya l', l'rabilu S. S., Cintterjee S. N., 1956. Secondiry sex-ratio in Indian cattle. $\%$. Tierz. ZuchlBiol., 68, 306-310.

13ı,ockUIs J., I957. The practical use of artificial insemination with goats. Tijdschr. siergeneesk, 82, 570$58 \mathrm{r}$, in Anim. Breed. Asbtr., 25, 1957.

BRANFORD R., 1957. Some breeding statistics. Agric. I. India., 12, 573-578.

Bi:eciI II. F., 1957. Intersuchungen über das verschobene Geschlechtsverhältnis, die Intersexualität und dic Fruchtbarkeit bei der Milchziegre. Tierz. Zutcht Biol., 69, 30-9o.

BuRGESS T. D., r953. The effect of month and season upon breeding efficiency obtained with artificial insemination. Canad. J. Agric. Sci., 33, 396-398.

ERB R. E.., WILBUR J. W., HiLtoN J.Il., J942. Seasonal variations in semen quality of the dairy bull. $J$. Dairy Sci., 25, 815-826.

IIAFEZ E. S. E., 1952. Conception rate in the buffalo. Rep. and Int. Congr. Physiol. Path. Anim. Reprod. Artit. Insem., 1, 97-100.

HaFez li. S. E., 1953. Conception rate and periodicity in the buffalo. Emp. J. Exp. Agric.,21, I5-21, in Anim. Breed. Abstr., 21, 1230.

Kushwaila N. S., Mukherjee D. P'., Bunttacinary P., i95. Seasonal variations in reaction time and semen qualities of buffalo bulls. Indian $J$. Vet. Sri., 25, 317-323.

MaKiIJANi II. K., I947. Cattle of Iurdia, Problem of mumbers. Indian liamn., 8, 365-372.

Mercier E., SAlisblirg G., I947. Scasonal variations in hours of daylight associated with fertility level of cattle under natural breeding conditions. J. Dairy Sci., 30, 747-756.

PATRIK T. E., I952. A study of seme factors affecting cfficiery of reproduction in dairy cattle serviced by

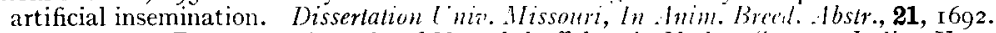

Pattabiraman D., r956. A study of Murrah buffaloes in Madras State. Indian Vet. J., 33, r88-204.

Prabiu S. S., Binattacharya P'., I953. Seasonal variations in conception rates in artificially breed cows. Indian J. Vet. Sci, 23, I29-133.

Setinski Z., Peticarie J., Kaciga M., i956. Artificial insemination in groats in Zagreb in 1952-1956. Vet. Gilas., 10, 906-910, in Anim. Breed. Absir., 26, 281.

SilnNMUGA SundaraM K. S., I95I. Birth rate among goats. Indian Vet. J., 34, I07-I I 7 .

\section{N.R.A.
BIBLIOTHEQUE UD $359 ?$
DOMAINE DE CROUELLE
63039 CLERMONT-FD CEDEX 2}

Imprimerie Bussiène à Saint-Amand (Cher), France. - 28-2-1962.

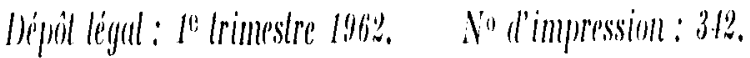

